\title{
August 2015 Critical Care Case of the Month: A Diagnostic Branch of Medicine
}

\author{
William T. Love, MD \\ Karen L. Swanson, DO \\ Department of Pulmonary Medicine \\ Mayo Clinic Arizona \\ Scottsdale, AZ
}

\section{History of Present IIIness}

A 66-year-old man had undergone an orthotopic heart transplantation on March 28th, 2015 due to end-stage cardiomyopathy. During a recent hospitalization from 6/26-7/2 a transbronchial lung biopsy was suggestive of subacute rejection. He was treated with:

- Plasmapheresis x 3

- Intravenous immunoglobulin (IVIG)

- 500 mg Solu-Medrol daily

- Tacrolimus held as supra-therapeutic level of 16.2

- Mycophenolate decreased to 500mg BID

- Prednisone at 10mg BID on discharge

On July 3rd he began having cough productive of clear sputum, nausea, vomiting, and headache. Subsequently he had body aches, subjective fever, chills, night sweats, and a poor appetite with a $4 \mathrm{~kg}$ weight loss over the last week. There was also a history of several falls after "losing his balance".

\section{Past Medical History}

There was also a history of type 2 diabetes mellitus, chronic kidney disease, coronary artery disease with coronary artery bypass grafting in 2000 .

\section{Physical Examination}

- Vital signs: T-37.1, HR-100, BP-130/88, RR-22, 96\% RA

- Heart: regular rate \& rhythm. 2/6 Systolic Murmur

- Lungs: clear to auscultation bilaterally

\section{Laboratory}

- Hemoglobin 9.7, WBC 6.3, creatinine 2.2, mildly elevated AST/ALT

- Lumbar Puncture- Protein 58 mg/dL, Glucose 46 mg/dL, 47 Nucleated cells

\section{Radiography}

A chest $\mathrm{x}$-ray was performed (Figure 1). 


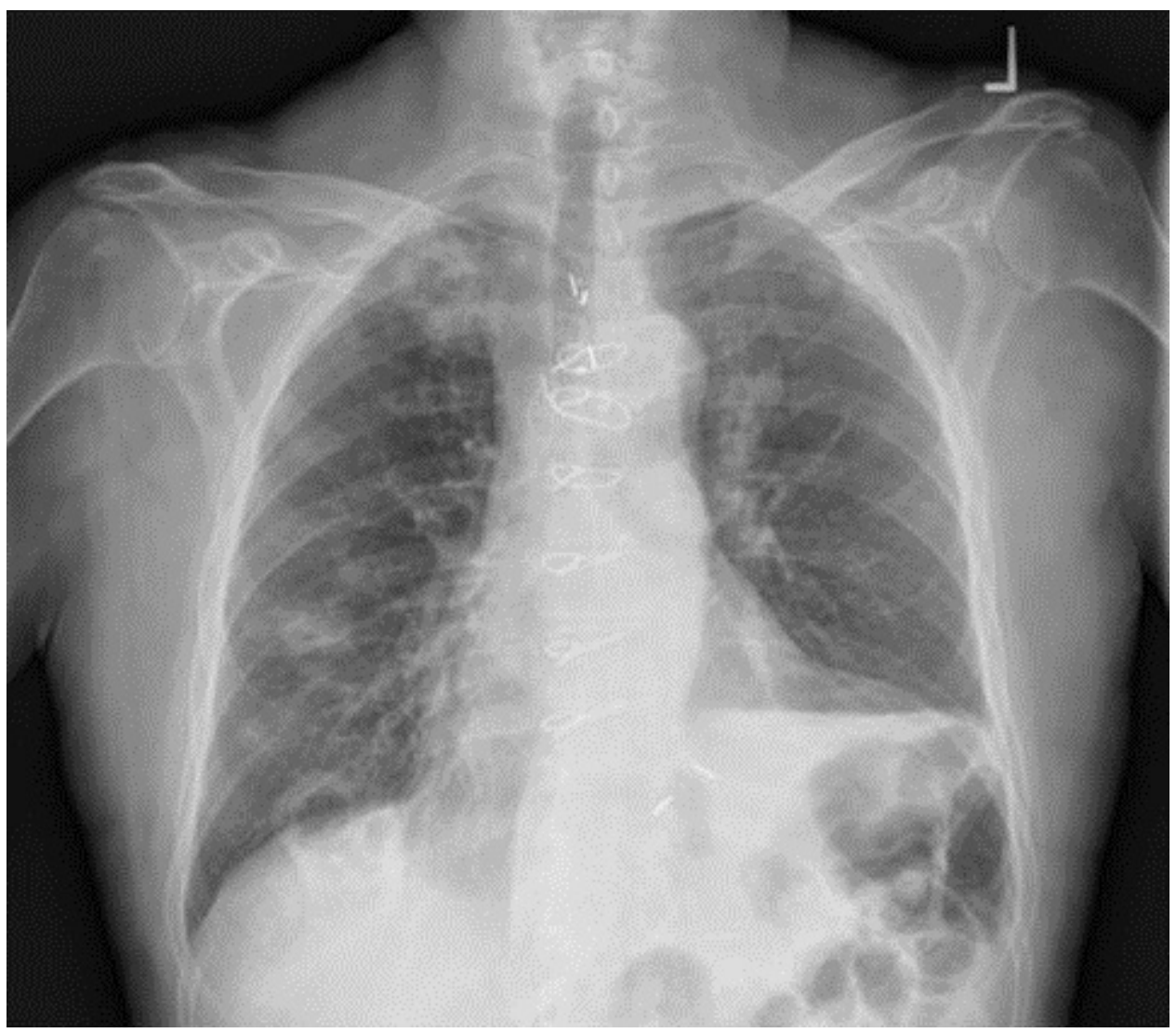

Figure 1. Admission PA of the chest.

Based on the chest x-ray and lumbar puncture, which of the following are true?

1. The chest $x$-ray and lumbar puncture findings in this clinical situation suggest cancer metastatic to the lung and brain

2. The chest $x$-ray and lumbar puncture findings in this clinical setting suggest an infection involving the lung and brain

3. The clinical findings suggest granulomatosis with polyangiitis (formerly known as Wegener's granulomatosis)

4. The clinical findings are suggestive of acute rejection

5. The clinical findings are suggestive of tuberculosis 


\section{Correct! \\ 2. The chest $x$-ray and lumbar puncture findings in this clinical setting suggest an infection involving the lung and brain}

The chest x-ray shows multiple cavitary masses and the protein and nucleated cell count are elevated on the lumbar puncture. There are many causes for these findings although in this clinical situation with an immunocompromised patient with apparent acute changes, the major consideration is an infectious process involving the lung and brain. Blood and sputum cultures were performed but were negative. To further investigate the patient's illness a thoracic CT scan (Figure 2) and MRI of the brain (Figure 3) were performed.

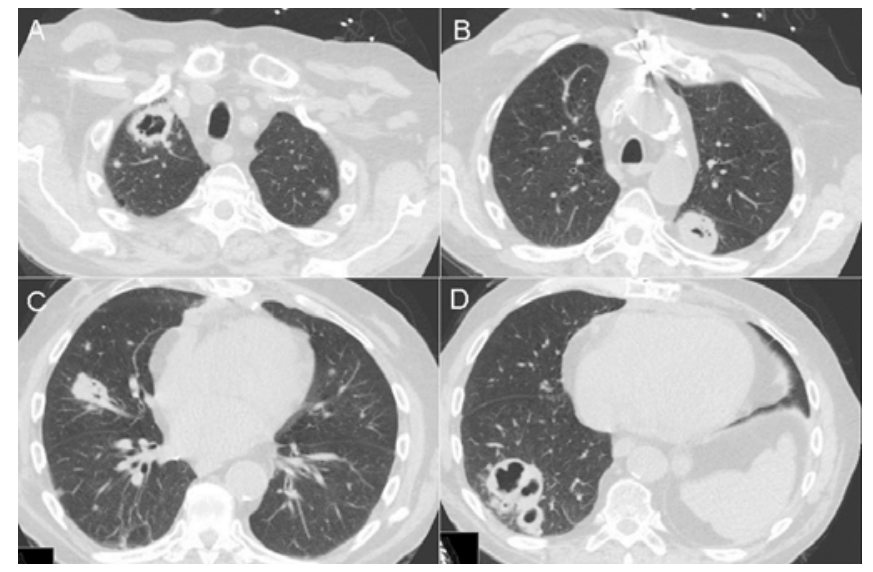

Figure 2. Representative views in lung windows from the thoracic CT scan.

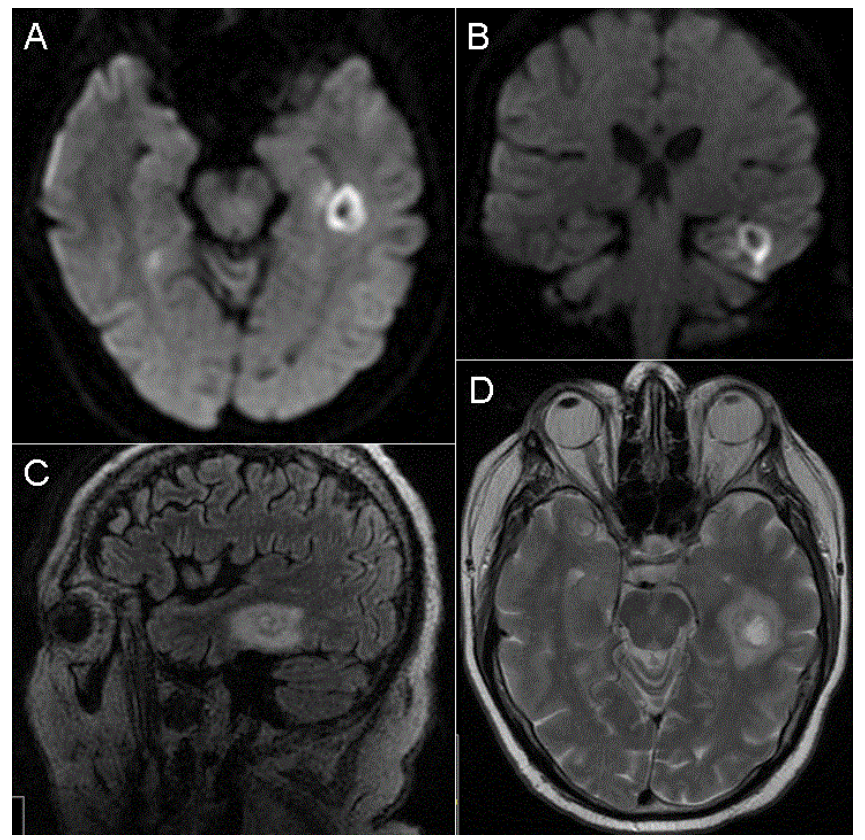

Figure 3. Representative images from the MRI of the brain. 
What should be done next to investigate the patient's probable infection?

1. Bronchoscopy with bronchoalveolar lavage (BAL) and/or transbronchial biopsy

2. Empirically begin antibiotics, anti-fungals and anti-tuberculosis medications

3. Increase the anti-rejection therapy

4. Needle biopsy of the brain

5. Video-assisted thorascopic lung biopsy (VATS) 


\section{Correct!}

\section{Bronchoscopy with bronchoalveolar lavage and/or transbronchial lung biopsy}

The differential diagnosis for cavitary lung disease is large (Table 1) (1).

Table 1. Differential diagnosis of cavitary lung disease

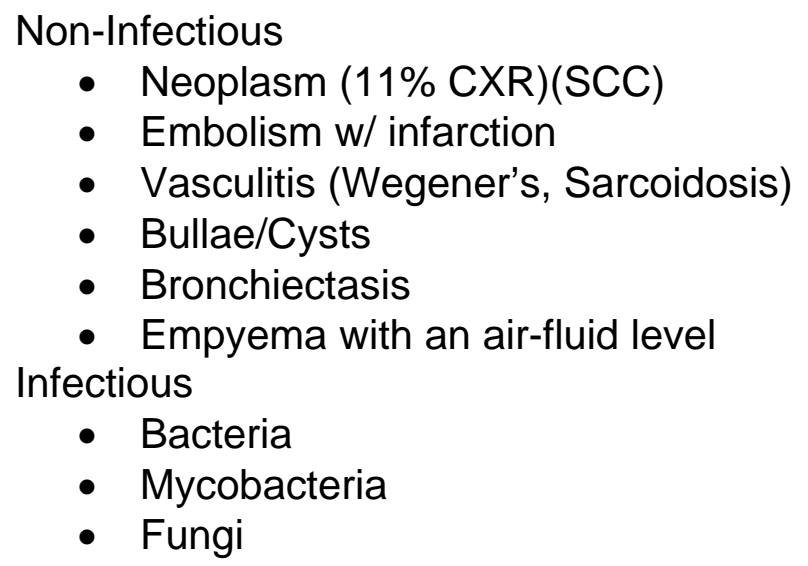

Many of these causes can also result in brain abscesses. Usually rejection does not cavitate. Because the differential is large, empiric therapy could be started but should be followed with efforts to establish a cause. Of the options listed, most would select a bronchoscopy as the lease invasive. Most favor aggressive pursuit of a diagnosis rather than empiric therapy in the immunocompromised host (1). Many pulmonary infections in this situation can be diagnosed with bronchoalveolar lavage. Aspergillosis with its characteristic $45^{\circ}$ branching chains (Figure 4) may be absent on BAL.

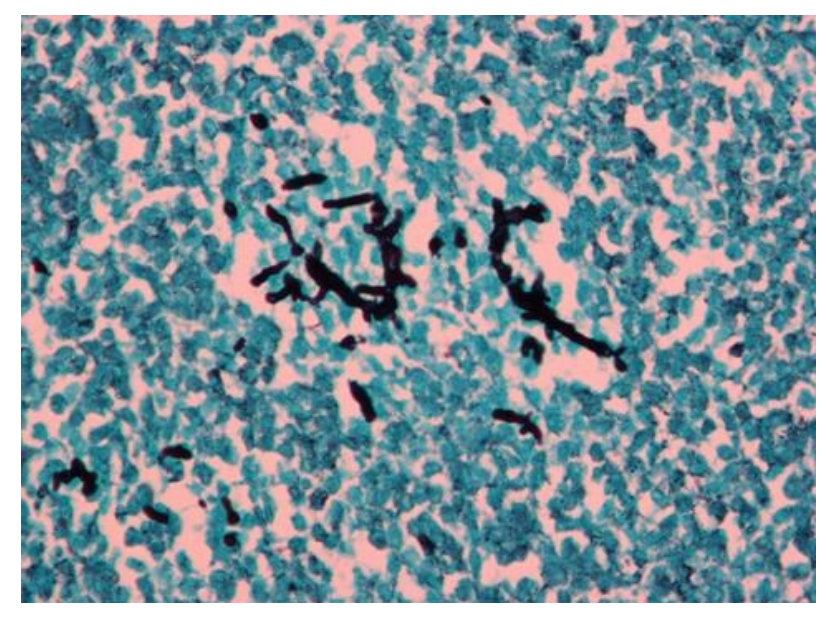

Figure 4. Silver stain showing numerous intraparencymal hyphae with acute angle branching consistent with invasive aspergillosis (From Shostak E, Liesching T, Wener

$\mathrm{K}$. A young woman with respiratory failure after a visit to compost station. American

Thoracic Society. Available at: http://www.thoracic.org/professionals/clinicalresources/clinical-cases/a-young-woman-with-respiratory-failure-after-a-visit-tocompost-station.php) 
A BAL with transbronchial biopsy was performed with the results as below:

- Pathology-- multiple small fragments of alveolated lung parenchyma with interstitial hemosiderin deposition. GMS stain negative for fungal organisms.

- Cytology--Right lower lobe bronchial lavage: Negative for malignancy. Fungal hyphae are present with morphologic features consistent with Aspergillus species.

- BAL with Aspergillus fumigatus, Aspergillus flavus and Pseudomonas (susceptible to all antimicrobials tested).

- BAL galactomannan antigen $>3.375$

- Serum galactomannan antigen $>3.375$ (normal $<0.5$ )

- Serum ß-D-glucan assay $>500 \mathrm{pg} / \mathrm{ml}$ (normal <60)

A presumptive diagnosis of invasive aspergillosis was made. Which of the following is the drug of choice for invasive aspergillosis?

1. Anidulafungin

2. Caspofungin

3. Lipid formulation of Amphotericin B

4. Micafungin

5. Voriconazole 


\section{Correct! \\ 5. Voriconazole}

First line treatment for invasive aspergillosis is now voriconazole (2). If the patient is intolerant or unresponsive to voriconazole, a lipid formulation of amphotericin $B$ may be added. An echinocandin (caspofungin, micafungin) may be added for 10-14 days before stepping down to monotherapy.

Our patient was treated with voriconazole and was improving.

\section{References}

1. Gadkowski LB, Stout JE. Cavitary pulmonary disease. Clin Microbiol Rev. 2008;21(2): 305-33. [CrossRef] [PubMed]

2. Walsh TJ, Anaissie EJ, Denning DW, Herbrecht R, Kontoyiannis DP, Marr KA, Morrison VA, Segal BH, Steinbach WJ, Stevens DA, van Burik JA, Wingard JR, Patterson TF; Infectious Diseases Society of America. Treatment of aspergillosis: clinical practice guidelines of the Infectious Diseases Society of America. Clin Infect Dis. 2008;46(3):327-60. [CrossRef] [PubMed] 